NUP-A-96-15

cond-mat/9611214

December, 1996

\title{
UNIVERSAL WIDE CORRELATORS IN NON-GAUSSIAN ORTHOGONAL, UNITARY AND SYMPLECTIC RANDOM MATRIX ENSEMBLES
}

\author{
Chigak Itoi 円 \\ Department of Physics, \\ College of Science and Technology, Nihon University, \\ Kanda Surugadai, Chiyoda-ku, Tokyo 101, Japan
}

\begin{abstract}
We calculate wide distance connected correlators in non-gaussian orthogonal, unitary and symplectic random matrix ensembles by solving the loop equation in the $1 / N$-expansion. The multi-level correlator is shown to be universal in large $N$ limit. We show the algorithm to obtain the connected correlator to an arbitrary order in the $1 / N$-expansion.
\end{abstract}

\footnotetext{
${ }^{1}$ e-mail: itoi@phys.cst.nihon-u.ac.jp
} 


\section{Introduction}

Recently, the universality of large $N$ (wide) connected correlator in random matrix theories is shown by Ambjørn, Jurkiewicz and Makeenko [1]. They solved the loop equation for the correlators with the motivation of studying the two dimensional gravity. The function form of the wide correlator does not depend on the details of the probability distribution of a matrix ensemble. The universality classification in terms of this correlator is also done in uniatry ensembles by Ambjørn and Akemann [2]. Brézin and Zee stressed this remarkably universal nature of random matrix theories from the view point of the physics in disordered systems [3]. Although some other universal quantities in random matrix theories have been well-known already [4], this new correlator can be calculated explicitly in various types of trace class ensembles in several approaches, and its universal nature can be observed manifestly [5].

In this paper, we calculate the large $N$ correlators in non-gaussian orthogonal and symplectic ensembles by solving the loop equation. Ambjørn, Chekhov, Kristjansen and Makeenko developed this method originally proposed in [四] to calculate the correlator in principle to an arbitrary order in $1 / N$-expansion in an arbitrary distribution of a unitary invariant ensemble [6]. Here, we extend this method to orthogonal and symplectic ensembles, which are well-known as useful description schemes for the level statistics of time reversal non-integrable quantum systems without and with spin degrees of freedom, respectively [4]. Calculation scheme for wide connected correlator to an arbitrary order in the $1 / N$-expansion have never been obtained in non-gaussian orthogonal and symplectic ensembles, though its leading order is calculated by solving an integral equation [7], its universality is shown by the super matrix method [8] and critical phenomena in an orthogonal ensemble have been studied by Brén and Neuberger as a model of two dimensional gravity on unoriented surface [9]. Here, we derive a loop equation of orthogonal and symplectic ensembles in the eigenvalue representation to calculate their wide connected correlator. We find a different nontrivial term from the unitary case in their loop equation. At the leading order in the $1 / N$-expansion, the extension of the solving scheme is straightforward, while at the next to leading order crucial modification is necessary. Here we give a general scheme to solve the loop equation up to an arbitrary order of the $1 / N$-expansion.

\section{The loop equation}

First we derive the loop equation for $\operatorname{orthogonal}(\beta=1)$, unitary $(\beta=2)$ and symplectic $(\beta=4)$ invariant ensembles. Their eigenvalue distribution is given by

$$
P\left(\lambda_{1}, \cdots, \lambda_{N}\right)=Z^{-1}\left|\Delta\left(\lambda_{1}, \cdots, \lambda_{N}\right)\right|^{\beta} \exp \left(-N \sum_{i=1}^{N} V\left(\lambda_{i}\right)\right),
$$

where $Z$ is normalization constant

$$
Z=\int_{-\infty}^{\infty} d \lambda_{1} \cdots \int_{-\infty}^{\infty} d \lambda_{N}\left|\Delta\left(\lambda_{1}, \cdots, \lambda_{N}\right)\right|^{\beta} \exp \left(-N \sum_{i=1}^{N} V\left(\lambda_{i}\right)\right)
$$


Van der Monde determinant is defined by

$$
\Delta\left(\lambda_{1}, \cdots, \lambda_{N}\right)=\prod_{1 \leq i<j \leq N}\left(\lambda_{i}-\lambda_{j}\right)
$$

and the potential is a polynomial $V(x)=\sum_{k=1}^{n} \frac{g_{k}}{k} x^{k}$. The Jacobian of an infinitesimal transformation of the integration variables $\lambda_{i}^{\prime}=\lambda_{i}+\frac{\epsilon}{z-\lambda_{i}}$ gives

$$
\begin{aligned}
& d^{N} \lambda^{\prime}\left|\Delta\left(\lambda_{1}^{\prime}, \cdots, \lambda_{N}^{\prime}\right)\right|^{\beta} \\
= & d^{N} \lambda\left|\Delta\left(\lambda_{1}, \cdots, \lambda_{N}\right)\right|^{\beta}\left(1+\epsilon\left(\frac{\beta}{2}\left(\sum_{i=1}^{N} \frac{1}{z-\lambda_{i}}\right)^{2}+\left(1-\frac{\beta}{2}\right) \sum_{i=1}^{N} \frac{1}{\left(z-\lambda_{i}\right)^{2}}\right)\right) .
\end{aligned}
$$

This transformation in the integral (2) gives the loop equation

$$
\frac{\beta}{2}\left\langle\left(\sum_{i=1}^{N} \frac{1}{z-\lambda_{i}}\right)^{2}\right\rangle+\left(\frac{\beta}{2}-1\right) \partial_{z}\left\langle\sum_{i=1}^{N} \frac{1}{z-\lambda_{i}}\right\rangle-N\left\langle\sum_{i=1}^{N} V^{\prime}\left(\lambda_{i}\right) \frac{1}{z-\lambda_{i}}\right\rangle=0
$$

where $\langle\cdots\rangle=\int d^{N} \lambda(\cdots) P\left(\lambda_{1}, \cdots, \lambda_{N}\right)$. Here, we define one point green function

$$
G(z)=\left\langle\frac{1}{N} \sum_{i=1}^{N} \frac{1}{z-\lambda_{i}}\right\rangle
$$

and $l$-level connected correlator

$$
G\left(z_{1}, \cdots, z_{l}\right)=N^{l-2}\left\langle\sum_{i_{1}=1}^{N} \frac{1}{z_{1}-\lambda_{i_{1}}} \cdots \sum_{i_{l}=1}^{N} \frac{1}{z_{l}-\lambda_{i_{l}}}\right\rangle_{c} .
$$

As discussed by Ambjørn, Chekhov Kristjansen and Makeenko [6], the loop equation (4) can be rewritten in terms of the one point green function and the connected two level corelator as the following closed form

$$
G(z)^{2}-\frac{2}{\beta} \oint_{C} \frac{d w}{2 \pi i} \frac{V^{\prime}(w)}{z-w} G(w)-\frac{1}{N}\left(\frac{2}{\beta}-1\right) \partial_{z} G(z)+\frac{1}{N^{2}} G(z, z)=0,
$$

where the contour $C$ encircles the singularities of $G(z)$. In the case of a finite $N$, these must consist of pole singularities, while in the $1 / N$-expansion these make up cuts. Here we assume the coefficient in an arbitrary order has cuts $\left(x_{1}, x_{2}\right), \cdots,\left(x_{2 s-1}, x_{2 s}\right)$. The third term in the left hand side in eq(5) is absence in the unitary case. This term requires a necessary modification of the $1 / N$-expansion

$$
G\left(z_{1}, \cdots, z_{l}\right)=\sum_{n=1}^{\infty} \frac{1}{N^{n}} G_{n}\left(z_{1}, \cdots, z_{l}\right) .
$$

Note that there is no odd degree term in the unitary ensemble. The coefficient $G_{n}(z)$ and $G_{n}(z, z)$ of an arbitrary order satisfies the following recursion relation

$$
\begin{aligned}
& n=0, \quad \hat{X} G_{0}(z)=0 \\
& n=1, \quad \hat{X} G_{1}(z)=\left(1-\frac{2}{\beta}\right) \partial_{z} G_{0}(z), \\
& n \geq 2, \quad \hat{X} G_{n}(z)=\sum_{m=1}^{n-1} G_{n-m}(z) G_{m}(z)+\left(1-\frac{2}{\beta}\right) \partial_{z} G_{n-1}(z)+G_{n-2}(z, z),
\end{aligned}
$$


where we define the operator $\hat{X}$ acting on an arbitrary function $f(z)$ by

$$
\hat{X} f(z) \equiv \frac{2}{\beta} \oint_{C} \frac{d w}{2 \pi i} \frac{V^{\prime}(w)}{z-w} f(w)-2 G_{0}(z) f(z) .
$$

We shall derive large $N$ correlators by solving this equation recursively.

\section{Large $N$ limit}

Here we obtain $G_{0}(z), G_{0}(z, w)$ and $G_{0}(p, q, r)$ in a non-gaussian ensemble. In the leading order $n=0$, the equation for $G_{0}$ can be written as

$$
\frac{\beta}{2} G_{0}(z)^{2}-V^{\prime}(z) G_{0}(z)-R(z, V)=0,
$$

where $R(z, V)$ is defined by

$$
\frac{1}{N}\left\langle\sum_{i=1}^{N} V^{\prime}\left(\lambda_{i}\right) \frac{1}{z-\lambda_{i}}\right\rangle=V^{\prime}(z) G(z)+R(z, V) .
$$

The quadratic equation (10) is solved with respect to $G_{0}$

$$
G_{0}(z)=\frac{1}{\beta}\left(V^{\prime}(z)-\sqrt{V^{\prime}(z)^{2}+2 \beta R(z, V)}\right) .
$$

If we assume s-cut solution, $G_{0}$ should have the form with the certain polynomial $M(z)$.

$$
G_{0}(z)=\frac{1}{\beta}\left(V^{\prime}(z)-M(z) \sqrt{\prod_{i=1}^{2 s}\left(z-x_{i}\right)}\right) .
$$

This assumption gives the following integral expression for $M$

$$
M(z)=\oint_{C_{\infty}} \frac{d w}{2 \pi i} \frac{1}{w-z} M(w)=\oint_{C_{\infty}} \frac{d w}{2 \pi i} \frac{V^{\prime}(w)-\beta G_{0}(w)}{(w-z) \sqrt{\prod_{i=1}^{2 s}\left(w-x_{i}\right)}}
$$

where the contour $C_{\infty}$ is a circle with the infinitely large radius. The second term of the integral vanishes due to the asymptotic behavior of $G_{0}(z) \sim 1 / z$ for $|z| \sim \infty$ and therefore

$$
M(z)=\oint_{C_{\infty}} \frac{d w}{2 \pi i} \frac{V^{\prime}(w)}{(w-z) \sqrt{\prod_{i=1}^{2 s}\left(w-x_{i}\right)}} .
$$

Substituting this into eq(12), one obtains

$$
\begin{aligned}
G_{0}(z) & =\frac{1}{\beta}\left(V^{\prime}(z)-\oint_{C_{\infty}} \frac{d w}{2 \pi i} \frac{V^{\prime}(w)}{w-z} \sqrt{\prod_{i=1}^{2 s} \frac{z-x_{i}}{w-x_{i}}}\right) \\
& =-\frac{1}{\beta} \oint_{C} \frac{d w}{2 \pi i} \frac{V^{\prime}(w)}{w-z} \sqrt{\prod_{i=1}^{2 s} \frac{z-x_{i}}{w-x_{i}}}
\end{aligned}
$$


The large $z$ asymptotics of $G_{0}$ in this expression gives

$$
\oint_{C} \frac{d w}{2 \pi i} \frac{w^{r} V^{\prime}(w)}{\sqrt{\prod_{i=1}^{2 s}\left(w-x_{i}\right)}}=\delta_{r s}, \quad r=0, \cdots, s .
$$

These equations and the arc independence of the chemical potencial

$$
0=\int_{x_{2 k}}^{x_{2 k+1}} d \lambda M(\lambda) \sqrt{\prod_{i=1}^{2 s}\left(\lambda-x_{i}\right)}, \quad k=1, \cdots, s-1,
$$

determine the location of the edges $x_{k},(k=1, \cdots, 2 s)$ of the cuts [10]. In the $s=1$ case, the edge values $x_{1}, x_{2}$ are determined only by the condition (15). Here we calculate a wide two level correlator in the $s=1$ case in the large $N$ leading order. The green function is obtained by acting the loop insertion operator

$$
\frac{d}{d V(w)} \equiv-\sum_{j=1}^{\infty} \frac{j}{w^{j+1}} \frac{d}{d g_{j}},
$$

on the free energy $F \equiv \frac{1}{N^{2}} \log Z$

$$
G(z)=\frac{d F}{d V(z)}+\frac{1}{z} .
$$

The $l(\geq 2)$ level correlator is obtained as well

$$
G\left(z_{1}, \cdots, z_{l}\right)=\frac{d}{d V\left(z_{1}\right)} \cdots \frac{d}{d V\left(z_{l}\right)} F .
$$

Thanks to the formula

$$
\frac{d V^{\prime}(z)}{d V(w)}=\frac{-1}{(z-w)^{2}},
$$

we can differentiate the one point green function $G_{0}(z)$ given by eq(14) to calculate the two level correlator in the leading order of the $1 / N$-expansion

$$
\begin{aligned}
G_{0}(p, q) & =-\frac{d}{d V(q)} \frac{1}{\beta} \oint_{C} \frac{d w}{2 \pi i} \frac{V^{\prime}(w)}{w-p} \sqrt{\prod_{i=1}^{2} \frac{p-x_{i}}{w-x_{i}}} \\
& =\frac{1}{\beta} \oint_{C} \frac{d w}{2 \pi i} \frac{-1}{(w-q)^{2}} \frac{1}{p-w} \sqrt{\frac{\left(p-x_{1}\right)\left(p-x_{2}\right)}{\left(w-x_{1}\right)\left(w-x_{2}\right)}} \\
& +\frac{1}{\beta} \oint_{C} \frac{d w}{2 \pi i} \frac{V^{\prime}(w)}{p-w} \sqrt{\frac{\left(p-x_{1}\right)\left(p-x_{2}\right)}{\left(w-x_{1}\right)\left(w-x_{2}\right)}} \frac{1}{2} \sum_{k=1}^{2 s}\left(\frac{1}{w-x_{k}}-\frac{1}{p-x_{k}}\right) \frac{d x_{k}}{d V(q)} \\
& =\frac{1}{\beta}\left[\frac{-1}{(p-q)^{2}}+\frac{\partial}{\partial q}\left(\frac{1}{p-q} \sqrt{\frac{\left(p-x_{1}\right)\left(p-x_{2}\right)}{\left(q-x_{1}\right)\left(q-x_{2}\right)}}\right)\right] \\
& +\frac{1}{\beta}\left[\frac{1}{2} M_{1}^{(1)} \frac{d x_{1}}{d V(q)} \sqrt{\frac{p-x_{2}}{p-x_{1}}}+\frac{1}{2} M_{2}^{(1)} \frac{d x_{2}}{d V(q)} \sqrt{\frac{p-x_{1}}{p-x_{2}}}\right]
\end{aligned}
$$


where the moment $M_{i}^{(k)}$ is defined by

$$
M_{i}^{(k)} \equiv \oint_{C} \frac{d w}{2 \pi i} \frac{V^{\prime}(w)}{\left(w-x_{i}\right)^{k} \sqrt{\left(w-x_{1}\right)\left(w-x_{2}\right)}} .
$$

In this single arc case, $M_{1}^{(1)} \frac{d x_{1}}{d V(q)}$ and $M_{2}^{(1)} \frac{d x_{2}}{d V(q)}$ is obtained only by differentiating the condition (15)

$$
\begin{aligned}
& M_{1}^{(1)} \frac{d x_{1}}{d V(q)}=\frac{1}{\left(q-x_{1}\right) \sqrt{\left(q-x_{1}\right)\left(q-x_{2}\right)}} \\
& M_{2}^{(1)} \frac{d x_{2}}{d V(q)}=\frac{1}{\left(q-x_{2}\right) \sqrt{\left(q-x_{1}\right)\left(q-x_{2}\right)}} .
\end{aligned}
$$

Substituting these into eq(20), we obtain the following expression of the wide two level correlator

$$
G_{0}(p, q)=\frac{p q-\left(x_{1}+x_{2}\right)(p+q) / 2+x_{1} x_{2}}{\beta(p-q)^{2} \sqrt{\left(p-x_{1}\right)\left(p-x_{2}\right)\left(q-x_{1}\right)\left(q-x_{2}\right)}}-\frac{1}{\beta(p-q)^{2}} .
$$

This result agrees with that in [四] in the unitary case $\beta=2$ and that in [7] for an arbitrary beta. We can calculate the three level correlator from this expression and the derivative of the edge values (21)

$G_{0}(p, q, r)=\frac{\left(x_{2}-x_{1}\right)\left[\left(p-x_{2}\right)\left(q-x_{2}\right)\left(r-x_{2}\right) / M_{1}^{(1)}-\left(p-x_{1}\right)\left(q-x_{1}\right)\left(r-x_{1}\right) / M_{2}^{(1)}\right]}{4 \beta\left[\left(p-x_{1}\right)\left(q-x_{1}\right)\left(r-x_{1}\right)\left(p-x_{2}\right)\left(q-x_{2}\right)\left(r-x_{2}\right)\right]^{3 / 2}}$

This result agrees with those obtained in [1] in the unitary case. It is obvious that the correlator of the level more than two takes a universal form, since the two level correlator depends on the distribution $V(\lambda)$ only through the edge values

\section{Gaussian ensemble}

Here we concern ourselves with the gaussian distribution. We calculate the one point green function up to second order in the $1 / N$-expansion. In the gaussian model, the one point green function and the wide two level correlator become

$$
\begin{aligned}
& G_{0}(z)=\frac{1}{\beta}\left(z-\sqrt{z^{2}-2 \beta}\right) \\
& G_{0}(z, w)=\frac{z w-2 \beta}{\beta(z-w)^{2} \sqrt{\left(z^{2}-2 \beta\right)\left(w^{2}-2 \beta\right)}}-\frac{1}{\beta(z-w)^{2}} .
\end{aligned}
$$

The loop equation (7) for the next order $G_{1}(z)$ is calculated by an reasonable assumption that the green function has no singularity outside the contour $C$ and the asymptotic behavior $G_{1}(z) \sim 1 / z^{2}$ for $|z| \sim \infty$ give

$$
\oint_{C} \frac{d w}{2 \pi i} \frac{w}{z-w} G_{1}(w)=z G_{1}(z),
$$


which yields the special solution of the loop equation (7)

$$
G_{1}(z)=\frac{1}{2}\left(1-\frac{2}{\beta}\right)\left(\frac{1}{\sqrt{z^{2}-2 \beta}}-\frac{z}{z^{2}-2 \beta}\right) .
$$

The general solution is given by adding the general solution of the homogeneous equation with respect to $G_{1}(z)$

$$
\frac{c_{1}}{\sqrt{z^{2}-2 \beta}}+\frac{c_{2} z}{\sqrt{z^{2}-2 \beta}}
$$

which does not satisfy the large $z$ asymptotics. Therefore the unique solution of eq(7) with the correct asymptotic behavior is given by eq(26). These results eq(24), eq( $(\overline{25})$ and eq(26) agree with those obtained by the replica trick [11.

The loop equation (8) for $G_{2}(z)$ is

$$
\hat{X} G_{2}(z)=\left(1-\frac{2}{\beta}\right) \partial_{z} G_{1}(z)+G_{1}(z)^{2}+G_{0}(z, z),
$$

The two level correlator with the coincident point can be defined as a finite function by limiting procedure

$$
G_{0}(z, z) \equiv \lim _{w \rightarrow z} G_{0}(z, w)=\frac{1}{\left(z^{2}-2 \beta\right)^{2}},
$$

which enables us to derive the solution of eq(27)

$$
G_{2}(z)=\frac{(2-\beta)^{2}\left(2 z^{2}+\beta-2 z \sqrt{z^{2}-2 \beta}\right)+2 \beta^{2}}{4 \beta\left(z^{2}-2 \beta\right)^{5 / 2}} .
$$

This is the unique solution of eq(27) as derived $G_{1}(z)$.

\section{$5 \quad$ Higher orders in the $1 / N$-expansion}

Now, we describe a general algorithm to solve eq(\$) to an arbitrary order in nongaussian case. In the orthogonal $\beta=1$ and symplectic $\beta=4$ cases, the derivative term $\partial_{z} G_{n-1}(z)$ in the right hand side of eq(7) and eq(8) requires the non-trivial modification of the algorithm in the unitary $\beta=2$ case. We have to solve the following type of equation for an unknown function $G(z)$ and a given function $F(z)$

$$
\hat{X} G(z)=F(z),
$$

We assume the regularity of $G(z), G_{0}(z)$ and $F(z)$ outside the region encircled by the contour $C$ and the large $z$ asymptotics of $G_{0}(z) \sim 1 / z$ and $G(z) \sim 1 / z^{2}$. A rewriting of the second term in the operation of $\hat{X}$ and a deformation of the contour $C_{z}$ to $C$ and $C_{\infty}$ give

$$
\begin{aligned}
G_{0}(z) G(z) & =\oint_{C_{z}} \frac{d w}{2 \pi i} \frac{G_{0}(w) G(w)}{w-z}=-\left(\oint_{C}+\oint_{C_{\infty}}\right) \frac{d w}{2 \pi i} \frac{G_{0}(w) G(w)}{w-z} \\
& =\oint_{C} \frac{d w}{2 \pi i} \frac{G_{0}(w) G(w)}{z-w} .
\end{aligned}
$$


The contribution from the contour integral of $C_{\infty}$ vanishes due to the large $z$ asymptotics of $G_{0}(z)$ and $G(z)$. Therefore the operation of $\hat{X}$ can be written as

$$
\hat{X} G(z)=2 \oint_{C} \frac{d w}{2 \pi i} \frac{M(w) \sqrt{\prod_{i=1}^{2 s}\left(w-x_{i}\right)}}{z-w} G(w) .
$$

In this expression of the operator $\hat{X}$, we can show a special solution of eq(29). If $M(z)^{-1}$ has no pole outside the region encircled the contour $C$, we find a special solution

$$
G^{S}(z)=\frac{F(z)}{M(z) \sqrt{\prod_{i=1}^{2 s}\left(z-x_{i}\right)}} .
$$

In general, $M(z)^{-1}$ has poles outside the contour $C$. We have to eliminate these unphysical singularities from the expression. Therefore, the solution becomes

$$
\begin{aligned}
G^{S}(z) & =\frac{F(z)}{2 M(z) \sqrt{\prod_{i=1}^{2 s}\left(z-x_{i}\right)}}-\oint_{C \cup C_{z}} \frac{d w}{4 \pi i} \frac{F(w)}{(w-z) M(w) \sqrt{\prod_{i=1}^{2 s}\left(z-x_{i}\right)}} \\
& =\oint_{C} \frac{d w}{4 \pi i} \frac{F(w)}{(z-w) M(w) \sqrt{\prod_{i=1}^{2 s}\left(z-x_{i}\right)}}
\end{aligned}
$$

which has no pole outside the contour $C$. Since the second term in the right hand side in eq(33)is annihilated by the action of the operator $\hat{X}$ with the expression eq(31), this $G^{S}(z)$ satisfies eq(29). General solution $G(z)$ of eq(29) is given by the linear combination of this special solution $G^{S}(z)$ and the allowed zero modes of the operator $\hat{X}$. In $s \geq 2$ case, Akemann shows that the allowed zero modes are

$$
\frac{z^{0}}{\sqrt{\prod_{i=1}^{2 s}\left(z-x_{i}\right)}}, \cdots, \frac{z^{s-2}}{\sqrt{\prod_{i=1}^{2 s}\left(z-x_{i}\right)}},
$$

which obey the boundary condition $G(z) \sim 1 / z^{2}(z \rightarrow \infty)$ 12. In $s=1$ case, the boundary condition excluded any zero mode to be added to $G^{S}(z)$. The unique solution of $G(z)$ as the green function is determined by the condition eq(18) that the green function which is the total derivative with respect to the loop insertion operator $\frac{d}{d V(z)}$. The equation (8) for $G_{n}(z)$ can be solved recursively in the above shown formula, and a correlator with an arbitrary level can be calculated to an arbitrary order in $1 / N$-expansion by acting the loop insersion operator eq(17) on the one point green function $G_{n}(z)$.

\section{Summary}

We have calculated two level and three level correlators in the large $N$ limit in nongaussian orthogonal, unitary and symplectic ensembles. We have obtained explicit forms of higher orders $G_{1}(z)$ and $G_{2}(z)$ in the gaussian ensemble. These results are consistent with those obtained in other method [11]. We have shown a new general algorithm to solve the loop equation recursively to calculate a correlator up to an arbitrary order in the $1 / N$-expansion for non-gaussian orthogonal and symplectic 
ensembles.

I would like to thank S. Higuchi, H. Mukaida and Y. Sakamoto for helpful discussions. I am grateful to S. Hikami for explaining his recent work. 


\section{References}

[1] J. Ambjørn, J. Jurkiewicz and Y.M. Makeenko, Phys.Lett.B251(1990)517.

[2] J. Ambjørn and G. Akemann, New universal spectral correlators, condmat/9606129.

[3] E. Brézin and A. Zee, Nucl.Phys. B402(1993)613.

[4] M. L. Metha, Random Matrices (Academic, New York, 1991).

[5] J. Ambjørn, C. F. Kristjansen and Yu. Makeenko, Mod.Phys.A7(1992)3187;

E. Brézin and A. Zee, Nucl.Phys. B441(1995)409;

J. D'anna, E. Brézin and A. Zee, Nucl. Phys.B443(1995)443;

E. Brézin and S. Hikami, Nucl PhysB479(1996)697

[6] J. Ambjørn, L. Chekhov, C. F. Kristjansen and Yu. Makeenko, Nucl.Phys. B404(1993)127.

[7] C. W. J. Beenakker Nucl.Phys B422(1994)515;

A. M. S. Macêdo and J. T. Chalker, Physical Review B 49 (1994)4695.

[8] G. Hackenbroich and H. A. Weidenmüller, Universality of Random-Matrix results for non-Gaussian ensembles, cond-mat/9412088.

[9] E. Brézin and H. Neuberger, Phys.Rev.Lett.65(1990)2098;

Nucl.Phys.B350(1991)513.

[10] J. Jurkiewicz, Phys.Lett.B245(1990)178.

[11] C. Itoi, H. Mukaida and Y. Sakamoto, Replica method for wide two level correlators in gaussian orthogonal, unitary and symplectic ensembles, NUP-A-96-16.

[12] G. Akemann, Higher genus correlators for the hermitian matrix model with multiple cuts, hep-th/9606004. 